\title{
Formation et identité syndicale à la CFE-CGC
}

L'entreprise comme espace de référence

Trade Union Identity and Training in the CFE-CGC. The Company as a Reference Model

\section{Corinne Delmas}

\section{OpenEdition \\ Journals}

Édition électronique

URL : http://journals.openedition.org/travailemploi/6775

DOI : 10.4000/travailemploi.6775

ISSN : $1775-416 \mathrm{X}$

\section{Éditeur}

DARES - Ministère du Travail

\section{Édition imprimée}

Date de publication : 1 octobre 2015

Pagination : $31-59$

ISSN : 0224-4365

Référence électronique

Corinne Delmas, « Formation et identité syndicale à la CFE-CGC », Travail et Emploi [En ligne],

144 | octobre-décembre 2015, mis en ligne le 01 octobre 2017, consulté le 30 avril 2019. URL : http:// journals.openedition.org/travailemploi/6775; DOI : 10.4000/travailemploi.6775 


\title{
Formation et identité syndicale à la CFE-CGC L'entreprise comme espace de référence
}

\author{
Corinne Delmas
}

\begin{abstract}
Instrument de transmission de valeurs, de savoir-faire, voire de savoir-être, la formation permet d'éclairer l'engagement syndical et la socialisation militante. Elle révèle également, en tant qu'outil de légitimation, les « effets de rhétorique » des syndicats, ainsi que le décalage entre les discours confédéraux et les pratiques. L'étude des stages proposés par la CFE-CGC montre les spécificités, l'activité et l'identité de cette organisation dans le paysage syndical français. De nature essentiellement pragmatique, les formations observées nous semblent constituer davantage un lieu de transmission de « savoir-faire » que d'acculturation militante. Face aux défis du renouvellement syndical et de la réforme de la représentativité, la CFE-CGC est confrontée à d'importants enjeux organisationnels de développement. La professionnalisation, l'essor d'un syndicalisme d'adhérents et l'émergence de la figure du manager attestent à la fois d'évolutions affectant l'ensemble de l'espace syndical et de la singularité d'un engagement ayant l'entreprise comme espace de référence.
\end{abstract}

I ssue de l'union en 1944 de plusieurs amicales et syndicats d'ingénieurs, chefs de services et techniciens, la Confédération générale des cadres (CGC) contribue depuis lors à faire exister le groupe des «cadres » (BOLTANSKI, 1982) en promouvant des institutions et formations spécifiques. Un Centre économique et social de perfectionnement des cadres et ingénieurs est ainsi créé dès 1951 par la Fédération nationale des syndicats d'ingénieurs et de cadres (FNSIC) ; la Fédération interprofessionnelle des agents de maîtrise, techniciens et assimilés (FIATA) inaugure quant à elle des centres analogues en 1956 afin de « faciliter l'adaptation des cadres moyens aux exigences modernes de l'économie productive de l'entreprise ${ }^{1} »$. Les formations prodiguées dans ces centres, parce qu'elles sont ouvertes à tous, se distinguent des formations

\footnotetext{
* Université Lille 2 ; Centre d'études et de recherches administratives, politiques et sociales (Ceraps) ; corinne. delmas@univ-lille2.fr.

1. Le Creuset, $\mathrm{n}^{\circ} 272,1957, \mathrm{p}$. 3. Les adhérents de la CGC ont à cette période une double affiliation, d'une part aux fédérations ou syndicats « professionnels » et, d'autre part, aux organismes interprofessionnels (FNSIC et FIATA) ou à la fédération des voyageurs représentants placiers (DELMAS, 2011).
} 
syndicales que la CGC met par la suite en place à destination des seuls syndiqués, « militants syndicaux [qui] ne sont pas des professionnels du syndicalisme ${ }^{2}$ », afin de les préparer aux mandats représentatifs.

Cette préparation a pris aujourd'hui une importance cruciale en raison de l'étiolement des viviers de socialisation militante et des transformations ayant affecté un syndicalisme devenu plus concurrentiel et technique (ETHUIN, Yon, 2010). Il s'agit d'une part d'outiller les militants confrontés à la technicité croissante de leurs mandats, à une intensification des négociations et à des thématiques parfois complexes (telles que les risques psychosociaux). Être crédibles face aux autres partenaires sociaux est essentiel pour des militants et des syndicats dont la légitimité se traduit de plus en plus par une expertise sur les conditions de travail, avec la montée en puissance des comités d'hygiène, de sécurité et des conditions de travail (CHSCT) (HENRY, 2012). D'autre part, la promotion professionnelle devient un enjeu majeur : la formation peut constituer un levier en la matière en s'inscrivant dans une démarche de valorisation des acquis d'une expérience syndicale que la loi de « rénovation de la démocratie sociale » de $2008^{3}$ a intégrée dans les négociations sur la gestion prévisionnelle des emplois et des compétences (GPEC).

Cette articulation entre deux formes d'engagement, syndical et professionnel, semble être particulièrement centrale à la $\mathrm{CFE}-\mathrm{CGC}^{4}$. Les notions de compétence et de responsabilité sont au cœur des principes de l'action syndicale de cette confédération ; elles correspondent à un syndicalisme visant conjointement les intérêts des salariés et de l'entreprise (BÉTHOuX et al., 2013) et y sont mises en avant depuis longtemps. Ses membres ont donc pu se les approprier (BOLTANSKI, 1982). Elles vont en outre de pair avec un « apolitisme », puis une « indépendance », que l'on peut analyser comme une forme de résistance à une dimension politique assimilée au conflit et qui permet de se démarquer d'une image de syndicat de droite qui lui est parfois accolée (GRUNBERG, MouriauX, 1979 ; BÉTHOuX et al., 2013).

Depuis les années 1990, la situation de la CFE-CGC a connu des changements : les cadres sont plus touchés par les plans sociaux qu'auparavant tandis que la CFDT la distance aux élections prud'homales de $1997^{5}$. Une décennie de « victoires défaites » pour le syndicalisme (Mouriaux, 2013, p. 102), les nouvelles formes de gestion sociale, le recul de l'engagement syndical, ainsi que les difficultés désormais rencontrées pour unifier un groupe « cadres » qui se serait diversifié (BOUFFARTIGUE et al., 2010 ; GROUX, 1990), banalisé (Bouffartigue, 2001) et dont les membres se penseraient de plus en plus comme des salariés à part entière (GROUX, 1983), sont autant de défis que la CFE-CGC doit relever. Par la suite, la réforme de la représentativité de 2008 provoque

2. Crépy (de) J., « La formation syndicale des cadres », Le Creuset, n 308, 2 octobre 1958, p. 27.

3. Loi n ${ }^{\circ} 2008-789$ du 20 août 2008 portant rénovation de la démocratie sociale et réforme du temps de travail.

4. Confédération française de l'encadrement - Confédération générale des cadres.

5. La CFE-CGC (21,9\% des voix) passe loin derrière la Confédération française démocratique du travail (CFDT ; $31,5 \%)$ dans le collège encadrement. Si la baisse est enrayée en 2002, l'écart persiste (22,8 \% contre 28,6 \%). La CFE-CGC retrouve la première place en 2008 (27,8 \% contre 22,9 \%) (BÉTHOuX. et al, 2013). 
des effets déstabilisateurs pour le syndicat, en débouchant sur une recomposition de ses fédérations et en provoquant des prises de position différenciées sur le maintien de la dimension catégorielle ${ }^{6}$.

Le maintien d'une stratégie de différenciation des cadres (BÉTHOUX et al., 2011) - qui s'appuie sur le diplôme, la durée du travail et la stabilité de l'emploi (AMossé, 2010 ; Amossé, Delteil, 2004 ; Bensoussan, 2010) - invite à interroger la formation comme courroie de transmission du sens que la confédération souhaite donner à son action. Outil de légitimation, elle peut donner à voir les « effets de rhétorique » du syndicat mais aussi les décalages entre les discours confédéraux et les pratiques (YoN, 2009 ; PERnOT, 2005). Outil de transmission de savoirs, de savoir-faire, de normes et de valeurs, elle apporte au chercheur des éléments de compréhension des ressorts de l'engagement syndical et de la socialisation militante des cadres. Des recherches sur les formations CFDT (MEYER, 1978 ; LESTIENNE, 1979 ; LoMBARDO, 2005), CGT (LOMBARDO, 2005) et $\mathrm{FO}^{7}$ (Yon, 2009) en ont montré les enjeux idéologiques. La formation nous semble de fait constituer une entrée pertinente pour étudier l'identité syndicale d'autant que chaque organisation syndicale a sa propre manière de concevoir l'engagement, qui se répercute nécessairement sur ses militants au moment des formations (NICOURD, 2009) ; on parle alors de « façonnage organisationnel du militantisme » (SAwicki, SimÉant, 2009, p. 19).

Nous nous proposons donc dans ce qui suit d'étudier la formation à la CFE-CGC comparativement à celles des autres organisations syndicales (voir encadré 1 « Sources, méthodologie et données de l'enquête »), mais aussi de saisir son évolution afin d'éclairer les dynamiques façonnant l'activité et l'engagement militants : dans un contexte marqué par le renouvellement générationnel et la réforme de la représentativité, on relève ainsi la montée en force du professionnalisme des formateurs en même temps que celle d'un syndicalisme d'adhérents et de la figure du manager-négociateur.

\section{ENCADRÉ 1}

\section{Sources, méthodologie et données de l'enquête}

Cet article mobilise des recherches sur les formations et l'expertise syndicales, réalisées sur plusieurs terrains (dont l'Observatoire du stress et des mobilités forcées de France Télécom) dans le cadre du projet ANR Formasynd (2007-2010) coordonné par Nathalie Ethuin et Karel Yon (Ceraps).

\footnotetext{
6. Sur les défis et effets contrastés de la loi n ${ }^{\circ} 2008-789$ du 20 août 2008 pour la CFE-CGC et ses fédérations en fonction du poids des non-cadres, cf. BÉTHOUX et al., 2013 ; BÉROUD, YoN, 2011. Si le projet de création d'une troisième force syndicale nationale, formulé par le président Bernard Van Craeynest, donne lieu à des tentatives de rapprochements avec l'Union nationale des syndicats autonomes (Unsa), l'idée de fusion provoque une levée de boucliers. Fin 2012, les statuts adoptés réaffirment la vocation de la confédération à « défendre l'intérêt des cadres ». La nouvelle présidente, Carole Couvert, rappelle à la fois la dimension catégorielle de la CFE-CGC lors de son $35^{\mathrm{e}}$ congrès, et le «pari du rassemblement » pris par la fédération énergie (partenariat avec l'Unsa pour les deuxième et troisième collèges).

7. Confédération française démocratique du travail ; Confédération générale du travail ; Force ouvrière.
} 
Nous avons consulté deux fonds d'archives : celui déposé à la Bibliothèque de documentation internationale contemporaine (BDIC ; Delta 1065) par l'un des fondateurs de la CGC, Roger Millot (Olivier, 2006) ; et celui de la Fédération des cadres de la fonction publique CGC, aux Archives nationales du monde du travail de Roubaix (ANMT, 100 AS 6-7).

Nous avons également dépouillé plusieurs publications : Ingénieurs et cadres de France de la Fédération nationale des syndicats d'ingénieurs et de cadres (FNSIC ; ${ }^{\circ} 33$, 1951-n 142, 1972), Maîtrise de France de la Fédération interprofessionnelle des agents de maîtrise, techniciens et assimilés FIATA ( $\left.\mathrm{n}^{\circ} 1,1955-\mathrm{n}^{\circ} 105,1990\right)$, Le Creuset. La voix des cadres $\left(\mathrm{n}^{\circ} 1,1944-\mathrm{n}^{\circ} 715,1977\right)$, Cadres et maîtrises (1977-1987) de la CGC, puis Encadrement magazine $\left(\mathrm{n}^{\circ} 1,1987-\mathrm{n}^{\circ} 202,2014\right)$ et la Lettre confédérale de la CFE-CGC.

De janvier 2008 à novembre 2010, nous avons observé dix stages interprofessionnels de formation syndicale au siège de la CFE-CGC, dont les thèmes étaient les suivants : ouverture sur le syndicalisme ; représentation du personnel ; élections professionnelles ; expression vocale et gestuelle ; argumentation ; comité d'entreprise ; négociation niveaux I et II ; créer et développer sa section syndicale ; manager son équipe syndicale. Nous avons observé en direct des manifestations organisées par la CFE-CGC (réunions, séminaires, congrès national de 2010) ; nous en avons visionné d'autres (congrès national de 2013) et suivi des stages et manifestations d'autres organisations de cadres (CFDT-Cadres,

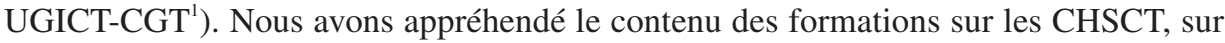
l'Europe et sur la lecture des comptes financiers grâce à l'analyse documentaire et aux entretiens que nous avons menés.

Enfin, nous avons réalisé vingt-cinq entretiens approfondis avec des responsables syndicaux, des formateurs, des salariés syndiqués et non syndiqués, des militants et des stagiaires de la formation syndicale. Ils s'ajoutent aux notes d'observation, aux échanges informels et aux données biographiques obtenues par les questionnaires recueillis à l'issue des cinq stages que nous avons suivis en 2008, mais aussi lors des tours de tables ou par les fiches « Restons en contact ${ }^{2} »$.

1. Union générale des ingénieurs, cadres et techniciens-CGT.

2. Distribuées en fin de stage par le formateur, ces fiches récapitulent les noms, les affiliations professionnelles et syndicales et les coordonnées de tous les participants, formateur(s) compris.

\section{Un syndicalisme qui fait la promotion du professionnalisme}

Les formations de la CFE-CGC, dont le nombre n'a cessé de croître, ont pour objectif de former des syndiqués performants. Pour ce faire, elles cherchent à renforcer chez les participants des aptitudes mobilisables aussi bien dans leur espace professionnel que personnel.

\section{Une offre croissante de formation}

La première session de formation est mise en place en 1960 à destination des seuls adhérents de la fédération de la métallurgie. À la suite de l'implantation et du 
renforcement de structures aussi bien géographiques (unions territoriales) que verticales (fédérations professionnelles) de la CGC, des sessions interprofessionnelles sont organisées qui doivent préparer à l'action des militants n'exerçant pas encore de responsabilités. Les formations syndicales plus poussées, destinées aux syndiqués titulaires de mandats, relèvent des sessions mises en place par les fédérations professionnelles. Les stages se diversifient ; outre ceux qui sont « réservés aux spécialistes d'un domaine précis [...] sur des sujets tels que les délégués du personnel, les comités d'entreprise, les conventions collectives ou les salariés ${ }^{8}$ », des sessions d'entraînement à la parole sont proposées ainsi que des stages de recherche et d'étude sur l'organisation des unions régionales ou les problèmes de Sécurité sociale par exemple. Un stage par mois est alors ouvert aux adhérents ; le contenu de l'offre de formation se stabilise à un moment où la CGC renforce sa place sur la scène sociale et dans les entreprises. En 1963, elle soutient les grèves de mineurs et participe à la mobilisation contre la politique de Neyrpic, entreprise de fabrication de grands équipements hydrauliques, à Grenoble. Tranchant avec des luttes syndicales souvent fondées sur des revendications quantitatives et exprimant le sentiment d'exclusion ressenti par de nombreux cadres, ce conflit de pouvoir s'inscrit dans l'affirmation d'un « syndicalisme de propositions » (Cornu, Groux, 2006, p. 18) qui s'appuie sur des ressources propres aux cadres, comme les savoirs de gestion. Par la suite, l'institution puis le renforcement d'une représentation spécifique des cadres au sein des comités d'entreprise (CE), par les lois du 18 juin 1966 et du 22 décembre 1972, ainsi que la concurrence d'autres organisations syndicales de cadres nouvellement créées (UGICT-CGT en 1963, UCC-CFDT en 1967, Ugica-CFTC en 1974, UCI-FO ${ }^{9}$ en 1977) incitent la CGC à mieux se saisir des revendications qui prennent forme dans les entreprises. Les enjeux sont donc majeurs au cours des années 1970, marquées par un souci d'ouverture à la jeunesse, aux cadres moyens et aux salariés intermédiaires ${ }^{10}$, et par l'attention portée à la formation, qui devient un thème récurrent de réflexion. Il s'agit de promouvoir une « école syndicale » de l'encadrement permettant au militant de se « perfectionner par des stages successifs $^{11} \gg$. Le besoin est d'autant plus important que les créations de sections

8. « $\mathrm{XVI}^{\mathrm{e}}$ congrès national de la CGC », Le Creuset, $\mathrm{n}^{\circ}$ 414, 20 juin 1963, p. 15.

9. Union confédérale des cadres CFDT ; Union générale des ingénieurs, cadres et assimilés - Confédération française des travailleurs chrétiens ; Union des cadres et ingénieurs-FO.

10. Les archives que nous avons consultées rendent compte de l'intérêt des fédérations et des syndiqués pour la formation syndicale et de leur méconnaissance de l'offre liée à la faible centralisation confédérale (ANMT, 100 AS 6, dossier 1, PV du 21 mars 1973 et du 23 janvier 1974), alors que les organisations syndicales sont de plus en plus en concurrence les unes avec les autres. Ce phénomène de concurrence est particulièrement perçu à la Fédération française des cadres des fonctions publiques de la CGC (FFCFP-CGC) ; un responsable y souligne la « montée des syndicats CFDT. Ils nous prennent des adhérents et sont présents dans les grands corps de l'État comme la Cour des comptes et l'ENA [École nationale d'administration]. Il faut compter maintenant avec une mentalité particulière de certains cadres qui adhèrent à la CFDT parce qu'ils considèrent être des salariés comme les autres » (ANMT, 100 AS 6, dossier 1, PV du 12 septembre 1973). La volonté d'ouverture aux salariés intermédiaires est importante dans certains secteurs comme la banque : elle se traduit par une « promotion sociale très large, pratiquement tous les employés ont vocation à devenir cadres. Dès lors, en les laissant adhérer aux syndicats de masse, le SNCB [Syndicat national des cadres de la banque] renonçait à la source essentielle de son recrutement » (ANMT, 100 AS 6, dossier 1, PV du 21 mars 1973). 11. Vilbenoît M., « Rapport sur la formation, XXIII ${ }^{\mathrm{e}}$ congrès de la CGC », Ingénieurs et cadres, n ${ }^{\circ}$ 167, 1977, p. 23. 
syndicales se multiplient, que le taux de syndicalisation reste faible et que les agents de maîtrise rencontrent des difficultés pour exercer leurs fonctions syndicales et participer aux instances confédérales ${ }^{12}$.

Au début des années 1980, l'unification confédérale se concrétise par les « états généraux de l'encadrement », la transformation de la CGC en CFE-CGC et une centralisation renforcée sous la présidence Marchelli : les fédérations interprofessionnelles laissent dorénavant place à des unions aux budgets réduits. Relevant désormais de la direction «Action » et constitué de neuf personnes, le Centre de formation syndicale (CFS) devient l'un des services les plus importants de la confédération. La CFE-CGC communique davantage sur son offre de formation et en publie les calendriers dans Cadres et maîtrise. Après que la loi n ${ }^{\circ} 82-915$ du 28 octobre 1982 (dite loi Auroux) relative au développement des institutions représentatives du personnel (IRP) prévoit des formations obligatoires pour permettre aux représentants du personnel d'exercer leurs attributions ${ }^{13}$, la CFE-CGC accroît son offre en conséquence. Elle crée le Centre d'études et de formation économique et social, association qui lance en 1983 des stages sur l'analyse financière, les mécanismes économiques et les orientations stratégiques de la confédération.

Des stages techniques (ouverture sur le syndicalisme, représentation du personnel, élections professionnelles, CE) coexistent avec des formations sur la dynamique de groupe, l'animation, l'expression et l'argumentation et, plus récemment, sur la négociation, le management d'équipe, etc. (voir encadré 2). Les objectifs affichés sont de former des « responsables », des « meneurs d'hommes », des « organisateurs », des « éducateurs », des « animateurs » des relations humaines et sociales, des « gestionnaires » et des « conseillers juridiques ».

\section{Le professionnalisme en tant que valeur distinctive}

Les formations ne semblent pas, aujourd'hui, s'appuyer sur un cadre idéologique fort. Seul le stage « Ouverture sur le syndicalisme » traite des « valeurs fondatrices de la CFE-CGC », parmi lesquelles l' « humanisme » qui, souligne le formateur, repose sur une vision de «l'homme au centre du système [...], ressource principale de l'entreprise $^{14} »$. La « liberté » est entendue « au sens de respect de la libre entreprise » et de « refus de tout déterminisme ». Le « professionnalisme » est mentionné comme marque de fabrique d'une CFE-CGC «à l'image de ses adhérents : responsable et combative, elle sait anticiper et prendre des initiatives ${ }^{15}$ ». Les notions d' "éthique » et de « responsabilité » sont pour leur part mobilisées lorsqu'il est question de la promotion de la « responsabilité sociale » de l'entreprise, du « développement durable »

12. XII ${ }^{\mathrm{e}}$ congrès de la FIATA, Cahiers de la maîtrise, $\mathrm{n}^{\mathrm{o}}$ 35, 1973, p. 11.

13. Formation économique des titulaires de comité d'entreprise (CE) ou des délégués du personnel (DP) selon les cas. 14. Verbatim recueillis dans le cadre de la formation «Ouverture sur le syndicalisme » (notes d'observation, janvier 2008).

15. Ibid. 
ENCADRÉ 2

L'offre confédérale de formation syndicale entre 2012 et 2015

\section{Module d'initiation}

Ouverture sur le syndicalisme

\section{Modules de perfectionnement}

Représentation du personnel dans l'entreprise ;

Le comité d'entreprise ;

Lectures des comptes et analyse financière ;

Le contrat de travail ;

Développer sa section syndicale ;

Les élections professionnelles dans l'entreprise.

Modules de développement des performances syndicales individuelles

Module 1 : Expression vocale et gestuelle;

Module 2 : Argumentation ;

Management de l'équipe syndicale ;

Communication écrite ;

Techniques de négociation niveau 1 ;

Techniques de négociation niveau 2 ;

Gérer les situations et les personnes difficiles ;

Négocier un accord de qualité de vie au travail (QVT) ;

Épargne salariale :

Module « Débutants »;

Module « Expérimentés ».

\section{Modules sur l'Europe}

Ouverture à l'Europe ;

Mise en place et fonctionnement des comités d'entreprise européens.

Sources : Encadrement magazine, $\mathrm{n}^{\mathrm{o}} 192,2012$ (calendrier $1^{\mathrm{er}}$ semestre 2013); $\mathrm{n}^{\mathrm{o}} 197,2013$ (calendrier $2^{\mathrm{e}}$ semestre 2013); $\mathrm{n}^{\mathrm{o}} 202,2014$ (calendrier $2^{\mathrm{e}}$ semestre 2014); CFS, Calendrier des sessions interprofessionnelles, $2^{\mathrm{e}}$ session 2015.

et de l'obtention, pour l'encadrement, d' « un droit à la reconnaissance et au respect à la mesure de leur devoir de compétence » : « il est de notre responsabilité de construire une éthique d'entreprise », ce qui constituerait une « petite évolution par rapport aux sempiternelles revendications salariales ». Le formateur conclut sur la « hiérarchie des fonctions et des salaires [...] fondée sur la compétence, la connaissance et la responsabilité » et ouverte « à tous, d'où l'importance de la GPEC [et de] l'investissement sur la formation professionnelle ». Explicables par l'histoire de cette confédération, par son syndicalisme de propositions et par l'importance de l'entreprise comme « espace de référence et d'action » (BÉTHOUX et al., 2013, p. 103), ces valeurs rappellent aussi celles 
qui sous-tendent les pratiques associatives ou la socialisation catholique des cadres, et qui expliquent leur « engagement incongru » au sens d'inapproprié et d'improbable chez ces « salariés de confiance » (GuILlaume, Pochic, 2009a, p. 535 ${ }^{16}$ ), proche à ce titre de celui, caritatif et humanitaire, de volontaires catholiques (SIMÉANT, 2009). Elles se rapprochent également des valeurs sur lesquelles s'appuie un « militantisme intimiste » (CASTELOT, 2004) qui ne s'affiche pas, par peur des rétorsions patronales, et s'exerce dans l'intimité de la section syndicale. Le formateur ne manque pas de préciser aux stagiaires : «Je ne vais pas vous endoctriner », puis insiste sur le réformisme par lequel la CFE-CGC se démarquerait des autres syndicats ainsi que sur son indépendance, y compris à l'égard des directions d'entreprise.

Les stagiaires montrent par leurs propos qu'ils apprécient la modération de la CFE-CGC et son rejet du dogmatisme ; ils opposent ce qu'ils considèrent être du pragmatisme au syndicalisme « archaïque » d'autres militants «campés sur des positions idéologiques » (cadre, secteur des télécommunications, syndiquée depuis trois ans) et d'organisations devant assumer un « héritage [...], un passé » (cadre, secteur des télécommunications, syndiqué depuis quatre ans). L' « autonomie » ou la « liberté », l'absence de « carcan » ou de « mainmise du syndicat » (cadre, secteur de l'énergie, syndiquée depuis deux ans), ainsi que le dialogue et la démocratie, sont présentés comme des spécificités cégécistes. En quête de formations très « concrètes », voire « terre à terre », afin d'aller « jusqu' au bout de leur mandat », ou de « devenir un expert » (élu CHSCT, secteur des télécommunications, adhérent depuis un an), les stagiaires souhaitent acquérir les techniques leur permettant de se présenter de manière à la fois professionnelle et neutre. Un responsable de formation dans son syndicat insiste ainsi sur l'importance de la crédibilité : «Quand on prend la parole, ou quand on argumente sur un dossier, ils savent que derrière c'est pas du vent [...]. On ne s'embarque pas à la légère » (manager ressources humaines, secteur des télécommunications, adhérent responsable de formation depuis trois ans). Si d'autres confédérations comme la CFDT cherchent à rendre leurs militants opérationnels par la formation, ce qui n'est pas sans susciter débats et critiques (GuILLAUME, 2011), un tel objectif est ici ancien, prégnant et davantage consensuel.

Le profil des formateurs, qu'ils soient salariés de la confédération ou prestataires externes $^{17}$, répond à cette quête de professionnalisme (DELMAS, 2011). Définie au niveau confédéral, l'offre de formation est organisée et assurée par la directrice du CFS et quatre formateurs qui, à la demande des fédérations et syndicats locaux, dispensent annuellement chacun une cinquantaine de formations, à Paris et en province, ce qui représente environ, pour l'année 2008, 220 stages sur une offre de 250. Claude CAMBUS

\footnotetext{
16. La relation privilégiée des cadres avec leurs employeurs implique une certaine loyauté et moins de motifs d'insatisfaction que les autres salariés. Leurs ressources et leur individualisme rendant le syndicat moins nécessaire à leur défense et les risques d'un engagement syndical pouvant mettre en péril leur propre carrière, leur engagement peut apparaître « incongru ». S'engager aujourd'hui dans le syndicalisme est tout sauf une évidence pour les salariés et $a$ fortiori pour les cadres (GuILlaume, Pochic, 2009a, p. 535).

17. Sous la présidence de Marc Vilbenoît dans les années 1990, des formateurs salariés de la confédération ont succédé aux permanents.
} 
(2003), secrétaire national en charge de la formation en 2003 et une responsable actuelle de la formation s'accordent pour considérer que la complexité des fonctions syndicales nécessite le recrutement de formateurs compétents « capables de répondre à des questions techniques ». La technicisation accrue des mandats et la valorisation d'un syndicalisme « responsable », de négociations et de propositions, justifient la mise en place de formations adéquates. Tous, stagiaires comme formateurs, insistent aujourd'hui sur le «professionnalisme » des formateurs : «Il y a vingt ans, des fois, on tombait sur des formateurs, c'était pas des pro. Maintenant, les formateurs qu'on a [...], c'est des pro » (manager ressources humaines, secteur des télécommunications, adhérent responsable de formation depuis trois ans) ${ }^{18}$.

Si l'ensemble des syndicats professionnalisent de plus en plus la manière dont ils abordent leurs différents domaines d'intervention en les confiant à des militants spécifiquement formés, cette tendance est davantage accentuée chez les syndicats réformistes. À l'instar de la CFE-CGC, les formations proposées par la CFDT (GuILLAUME, 2011) témoignent ainsi de la valorisation d'une « logique compétences » qui entend répondre à plusieurs besoins et tendances : la complexification des fonctions syndicales, la promotion d'un syndicalisme de propositions, l'essor des négociations (cf. supra); mais aussi les attentes de cadres abordant l'activité syndicale avec les mêmes exigences de «professionnalisme » que dans leur métier, les difficultés rencontrées par des non-cadres pour occuper une place de «leader» ou les anticipations de sorties du syndicalisme par des permanents dont la « carrière » syndicale est menacée par la loi de 2008. Contrairement à la CFDT ou à la CGT, la CFE-CGC ne propose pas ou peu de formations nettement orientées vers l'acquisition d'une « culture d'organisation » et militante. Dans un contexte de fort renouvellement de ses responsables, la CFDT a resserré son offre de formation sur des questions d'organisation interne et de transmission d'une « bonne vision » des choses s'appuyant sur le cadre réformiste (Guillaume, 2011, p. 117). À la CGT, l'éducation syndicale vise à transmettre les « fondamentaux » de la lutte des classes et à développer les capacités critiques (ETHUIN, SiBLOT, 2011, pp. 66-69) ${ }^{19}$.

Les formateurs de la CFE-CGC, quant à eux, mettent en avant leur statut de salarié (et non de syndiqué) comme gage de neutralité. Ils se présentent et sont perçus comme des « experts juristes » et des « porte-paroles » de la confédération, utilisant à la fois le « nous » et le « vous » (voir encadré 3 ).

Ce positionnement suscite parfois la surprise, voire l'incompréhension, chez les stagiaires participant au stage « Ouverture au syndicalisme » destiné aux nouveaux

\footnotetext{
18. Si elles ne permettent pas d'objectiver cette absence de professionnalisme, les archives rendent compte des besoins en formateurs de certaines fédérations, dont la fédération des cadres de la fonction publique (ANMT, 100 AS 6, dossier 1, PV comité fédéral du 22 janvier 1973).

19. Personnellement, nous avons pu constater ce souci de développement des capacités critiques et d'outillage théorique dans l'offre de formation à destination des cadres et agents de maîtrise, en particulier dans deux stages de l'UGICT-CGT observés en 2007, portant sur les inégalités entre hommes et femmes, organisés à l'Institut des sciences sociales du travail (ISST) de Paris 1.
} 


\section{ENCADRÉ 3}

\section{Le positionnement de « professionnel » salarié d'un formateur}

Le formateur, dans sa présentation du «professionnalisme » comme «valeur fondatrice de la CFE-CGC », évoque son statut de salarié à la CFE-CGC, non syndiqué. Un stagiaire s'enquiert : «Il n'existe pas de syndicat ici [à la confédération CFE-CGC] ? » Le formateur précise que « si », mais qu' « il n'y a pas de RP [représentants du personnel] car personne ne s'est présenté ». Face à l'étonnement croissant de son auditoire, il souligne la « relation de subordination à Van Craeynest » et le rôle central des experts, dont il fait partie, comme acteurs du syndicalisme et forces de proposition : « Nous les experts, on est à la base, parfois, des propositions » (notes d'observation, stage « Ouverture sur le syndicalisme », 2008).

adhérents. Une chargée d'études salariée de la confédération souligne ainsi combien « la position de formateur est un peu difficile parfois [...] ; il faut leur dire que nous sommes salariés [...] [et qu']on va pas vous donner notre point de vue, parce que ça n'a aucun intérêt ».

À l'occasion, l'accent est mis sur l'importance du droit comme outil syndical ; ainsi, l'animateur du stage $\mathrm{CE}$, prestataire d'un cabinet de conseil et d'expertise auprès des IRP, met en avant son statut de juriste et souligne combien « on se retrouve toujours à se retourner vers les règles écrites, les règles de droit » dans un contexte marqué par l'essor des négociations collectives, la « juridicisation des rapports sociaux » et la « nécessaire soumission des organisations syndicales à la rationalité juridique » (WILlEMEZ, 2003, p. 25). Le droit doit également contribuer à la « réassurance » des stagiaires face à l'équipe de direction. Ce discours trouve un certain écho chez des militants, dont certains soulignent la complexité du « métier de syndicaliste » où « il faut toujours se mettre à niveau » (cadre, secteur bancaire, permanent titulaire de nombreux mandats, syndiqué à la CFE-CGC depuis douze ans) en raison du rythme soutenu des négociations : « il y a toujours un accord en cours », souligne une permanente syndicale du secteur bancaire syndiquée depuis trente ans. Une cadre du secteur des télécommunications, déléguée du personnel (DP) et suppléante $\mathrm{CE}$, syndiquée à la CFE-CGC depuis trois ans, insiste sur la nécessité d'être « professionnelle » face à des « RH [ressources humaines qui] nous envoient par exemple des réponses à nos questions hors délai ».

Les militants font parfois face à des risques de rétorsion, voire de discrimination : « J'ai postulé à l'époque $[. .$.$] à ce poste, [. .$.$] je n'ai pas été retenue. ... Je... sens que$ mon patron actuel [...] a toujours mélangé en moi le [côté] syndicaliste et le [côté] financier » (cadre du secteur de l'énergie, DP, syndiquée depuis quatre ans). Loin d'être propres aux adhérents CFE-CGC, les blocages de carrière sont souvent mentionnés dans le cadre de l'enquête REPONSE (Relations professionnelles et négociations d'entreprise) (PERNOT, Pignoni, 2008). Mais ils sont alors liés au recours croissant 
à la grève, qui ne constitue pas le répertoire d'action privilégié à la CFE-CGC; de nombreux stagiaires ont explicitement rejeté grèves et manifestations. Par ailleurs, les femmes et les ouvriers semblent plus exposés que les hommes occupant une position d'encadrement.

La perception de ces risques n'en présente que plus d'intérêt, attestant en creux des spécificités de l'engagement à la CFE-CGC. Pour certains syndicalistes, elle semble aller de pair avec un ton plus revendicatif, peut-être en réaction à la critique récurrente d'un positionnement pro-patronal. Pour d'autres, les craintes de blocage de carrière sont ressenties comme injustes, et ce d'autant plus que l'engagement est vécu comme devant « viser conjointement les intérêts des salariés et ceux de l'entreprise (portés par la direction) » (BÉTHOUX et al, 2013, p. 108). Cette perception de l'engagement, alliée à des enjeux individuels de promotion professionnelle pour des non-cadres aspirant à se faire « repérer » comme des salariés auxquels des responsabilités pourraient être confiées, peuvent engendrer une dissonance entre le sens donné à l'action et la réalité vécue. Mais pour une dernière partie des syndicalistes, ces blocages sont moins perçus en termes de rétorsion patronale à l'action syndicale que comme liés à un moindre investissement professionnel :

« À partir du moment où vous avez un mandat social [...], vous avez des heures de délégation [...] ; il faut faire les deux, donc votre collègue lambda qui n'a pas ce rôle va travailler, on va dire sur son opérationnel 35 heures et vous, vous allez travailler, ben $x$ d'heures. Donc il est clair que ça pose un souci. [...] C'est par ce biais-là que ça peut être pénalisant. »

(Cadre, DP et trésorière CE, secteur de l'énergie.)

Investis au travail, voire privilégiant leur carrière, certains stagiaires indiquent d'ailleurs ne pas prendre toutes leurs heures de délégation ou recourir aux RTT pour suivre les formations.

\section{Quand les engagements professionnel et syndical s'entremêlent}

Articuler engagement militant et carrière professionnelle est une préoccupation forte chez des stagiaires qui sont majoritairement des cadres ou assimilés du secteur privé, secteur qui compte peu de syndiqués, comparativement au secteur public ${ }^{20}$. Plusieurs d'entre eux ont un niveau bac +5 , notamment parmi les participants aux stages ouverture, expression vocale et gestuelle ou argumentation (voir annexes, tableau 2). Près d'un tiers des stagiaires sont titulaires d'un diplôme de niveau III (brevet de technicien supérieur [BTS] ou diplôme universitaire de technologie [DUT]) et un peu moins d'un quart d'un diplôme de niveau II (principalement d'ingénieur) : ces proportions attestent la percée des filières techniques dans l'entreprise et le poids

20. On estime à 7,5\% la proportion des ingénieurs et cadres du privé qui déclarent appartenir à un groupe syndical ou professionnel, contre $25 \%$ dans le public (AMOssé, 2004). 
croissant des titulaires de BTS-DUT au sein de l'encadrement ${ }^{21}$; les formations initiales des cadres demeurent toutefois diverses. Les participants au stage «Élections professionnelles » que nous avons observé sont des syndicalistes à forte ancienneté, très engagés, et comptent parmi eux un tiers de permanents (voir annexes, tableau 3); certains sont des salariés non-bacheliers tandis qu'une majorité a au maximum un niveau III de formation initiale (voir annexes, tableau 2). Ce profil mixte, pour moitié techniciens et agents de maîtrise, pour moitié cadres, majoritairement commerciaux, soulève la question des enjeux professionnels de l'adhésion à cette confédération - dont l'accès au statut cadre - et de l'engagement militant différencié des cadres et non-cadres (BÉTHOUX et al., 2013). Dans le secteur des assurances, dont sont issus de nombreux participants au stage «Élections professionnelles » (voir annexes, tableau 4), nous avons noté que les cadres ont un engagement syndical plus grand que les agents de maîtrise et techniciens, ce dont témoigne leur propension à cumuler les mandats.

L'ancienneté syndicale est, tous stages confondus, assez faible (deux ans en moyenne) (voir annexes, tableau 3). Si le stage " Ouverture sur le syndicalisme » s'adresse aux nouveaux adhérents, ceux-ci sont très présents dans la plupart des formations. Les femmes sont parfois aussi nombreuses que les hommes, voire majoritaires (voir annexes, tableau 1), alors qu'elles sont minoritaires au sein de la CFE-CGC ${ }^{22}$. Dans les stages suivis par une majorité de femmes, les syndicalistes cumulent moins de deux mandats en moyenne et ont peu d'ancienneté (moins de quatre ans) (voir annexes, tableau 1). Le stage consacré aux élections professionnelles s'avère le moins féminisé ( $1 \%$ de femmes) et compte au contraire des militants à forte ancienneté (six ans et demi en moyenne) (voir annexes, tableau 3) et cumulant plus de quatre mandats chacun (voir annexes, tableau 1). Les stages que nous avons observés nous ont permis de constater tout à la fois une arrivée récente de femmes à la CFE-CGC, qui ont moins de mandats et des décharges horaires pour exercer leurs mandats plus limitées que les hommes ${ }^{23}$.

Certes, il existe quelques contre-exemples - ainsi cette militante depuis trente ans, devenue permanente de son syndicat, après avoir « occupé un peu tous les mandats ». Mais on peut noter qu'elle est agent d'une banque du secteur public ${ }^{24}$, dont le taux de

21. Ils sont $18 \%$ en 2008, contre $12 \%$ en 1985 ; ce sont les plus représentés parmi les cadres issus de la promotion interne (36\%) (CADET, MÖBUS, 2011).

22. D'après SILVERA (2006) : 18,5\% des adhérents de la CFE-CGC sont, en 2002, des femmes, contre $28 \%$ à la CGT, $40 \%$ à FO, $44 \%$ à la CFDT et $46 \%$ à la CFTC; elles constitueraient un quart des adhérents selon les chiffres de la confédération en 2008. D'après un récent rapport du Conseil économique, social et environnemental s'appuyant sur l'enquête annuelle de la Confédération européenne des syndicats, 29,1\% des adhérents de la CFE-CGC sont, en 2014, des femmes, contre 36,8 \% des adhérents de la CGT, $47 \%$ de ceux de la CFDT, $45 \%$ de ceux de la CGT-FO, $42 \%$ de ceux de la CFTC et $52 \%$ de ceux de 1'Unsa (Guichet, 2015, p. 23).

23. Ce constat conforte celui du faible poids des déléguées au congrès de la CFE-CGC de 2010, qui comptait moins de $15 \%$ de femmes, contre $25 \%$ pour les autres confédérations (BÉTHOuX et al., 2013), voire $38 \%$ au congrès de la CFDT (Guillaume, Pochic, 2009a) ; concernant la CFE-CGC, BÉTHoux et al. (2013) ont estimé à $45 \%$ le taux de femmes actives détenant plus de deux mandats, avec une décharge d'activités professionnelles pour exercer leurs mandats alors plus limitée, et à $54 \%$ celui des hommes.

24. Seuls 4 stagiaires, parmi les 71 dont le statut est connu, relèvent du secteur public (en tant que fonctionnaires d'une entreprise privatisée ou agent d'une banque du secteur public) ; ce faible nombre s'explique par les formations spécifiques dont bénéficient les fonctionnaires, qui sont proposées par la fédération des fonctions publiques CFE-CGC. 


\section{ENCADRÉ 4}

\section{Une trajectoire exemplaire}

M. me reçoit au siège de son syndicat. Elle a adhéré à la CFE-CGC dès son recrutement, « très naturellement parce qu'en fait quand on rentre à la banque, les syndicats, disons, font un pot, nous accueillent ». Elle opte pour la CGC « en tant que cadre », attirée par le «côté catégoriel »: «Je me suis dit : "Je vais me retrouver avec mes pairs [...] et je pense que je serai plus à l'aise." » Parvenue trente ans plus tard au faîte de sa carrière, après avoir « occupé un peu tous les mandats » au sein de son syndicat, sollicitée lors des élections, elle perçoit cette responsabilité dirigeante et le statut de permanente comme une « opportunité » à un moment où son avancement de carrière est bloqué et où elle « cherche à faire autre chose » : « personnellement j' ai atteint mon bâton de maréchal, depuis déjà un certain temps, et c'est d'ailleurs un des points faibles à la banque, c'est que, atteignant son bâton de maréchal à un âge relativement jeune, autour de 45 ans, il reste encore vingt ans. Dans l'évolution de la banque actuellement, je me suis beaucoup interrogée en me disant : "Bon moi, ça ne me convient pas" ». Cette situation motive sa candidature à la permanence de son syndicat. Le mandat de secrétaire générale adjointe, assimilé à un « poste », lui permet d'exercer des activités nouvelles, en lien avec ses compétences professionnelles, sa formation universitaire en économie et gestion des entreprises, et ses fonctions antérieures de « risk manager » : «C'était plus par rapport à un questionnement sur la gestion RH de la banque, c'est ce qui m'a conduit au poste que j'occupe maintenant. » $\mathrm{M}$. insiste sur son engagement professionnel : «On a d'abord son travail et [ensuite] la fonction qu' on occupe en tant qu'élu CE ou dans les IRP [...] ; on la fait, je dirais, de manière très, très accessoire et très distante, enfin, en tout cas, les gens élus CFE-CGC, parce qu'on est d'abord cadres, on a très peu de temps à consacrer à ce genre de choses [...]. Après on fait comme on peut sur le terrain, voilà. Alors qu'en venant ici justement, j' ai découvert plus avant, je dirais, les enjeux... des IRP [...] ; les permanents, qui ont du temps, c'est leur job, et donc forcément ils s'y investissent. » M. ne souhaite pas se représenter car elle craint de ne plus être au niveau, d'être en situation de décrochage par rapport à ses anciennes fonctions : «Nous, en tant que cadres, pour se recaser après, quand on a décroché pendant trois ans, faut déjà s'y remettre, les choses évoluent tellement vite. [...] Quand vous avez encore quinze ans à travailler, vous ne pouvez pas vous permettre. » Se demandant s'il est pertinent pour elle de « rester sur Paris, dans une direction générale » et comment se maintenir dans la course alors qu' « une [nouvelle] génération arrive », M. a réalisé un bilan de compétences, et prépare dans la foulée un master 2 en ressources humaines/responsabilité sociale des entreprises, comptant sur cette formation « pour [s]e trouver un point de chute».

syndicalisation est plus élevé que dans le privé. Sa trajectoire atypique nous semble particulièrement bien rendre compte de l'enchâssement professionnel de l'engagement syndical. En effet, les mandats mobilisent des compétences de métier et sont perçus comme inhérents à un engagement professionnel qui reste prioritaire (voir encadré 4).

Allant de pair avec l'investissement professionnel, l'engagement syndical peut être motivé par la volonté de connaître la politique de l'entreprise. C'est ce qu'indique un 
délégué syndical (DS) alors membre du CHSCT, adhérent à la CFE-CGC depuis trois ans : «La première fois que je me suis présenté à une élection au comité d'entreprise, c'est parce que, au niveau où j'étais, je n'avais pas d'information. » Une autre explique qu'elle s'est syndiquée parce qu'elle souhaitait obtenir des informations que seuls les syndiqués peuvent avoir. Un contrôleur interne, trésorier du CE, remarque : « Le nombre de fois où j'ai appris des choses en CE qui m'ont servi pour mon travail ! » Un cadre du secteur bancaire, permanent syndical, souligne que c'est son engagement syndical qui lui a permis d'avoir une « vision sur les comptes de l'entreprise, [...] une meilleure connaissance de l'organisation, [...] une vue beaucoup plus large des problèmes ».

Souvent, dans leurs propos, les stagiaires mélangent formations syndicales et formations professionnelles, montrant ainsi qu'ils les considèrent comme complémentaires, voire interchangeables. Plusieurs ont suivi des formations à des fins, disent-ils, d' « information personnelle » : un agent de maîtrise des télécommunications, élu CHSCT, indique par exemple avoir suivi le stage « Lecture des comptes financiers » « en formation personnelle». Les responsables de stages précisent vouloir « réconcilier les deux », la formation devant « servir dans tous les domaines ».

\section{La reconversion professionnelle des militants}

Selon un responsable syndical de formation, l'un des enjeux majeurs de la formation est de « permettre d'entrer dans le syndicalisme, mais également d'en sortir ». Commune à l'ensemble des organisations syndicales, cette préoccupation se traduit par des actions diverses, notamment de formation professionnelle, à destination des permanents ${ }^{25}$.

À la CFE-CGC, l'objectif est que « les militants puissent rendre à l'entreprise l'expérience acquise en tant que "managers" syndicau ${ }^{26} »$. À cette fin, une observation de l'activité militante de vingt conseillers prud'hommes est lancée en 2006 alors que d'autres syndicats cherchent également à valoriser les acquis militants ${ }^{27}$. À l'issue de cette observation réalisée par une équipe de chercheurs du Conservatoire national des arts et métiers, dirigée parYves Clot, sont mis en exergue plusieurs « conflits inhérents au travail des militants de la CFE-CGC », tels que le dilemme entre loyauté envers l'employeur ou loyauté envers le syndicat, ou la question d' « être d'abord syndicaliste ou d'abord professionnel ?» (TomÀs et al., 2014, pp. 184-185). Les conclusions de cette observation ont permis l'élaboration d'un référentiel d'activités qui recense et décrit les compétences mobilisées par les militants dans le cadre de leur activité

\footnotetext{
25. Un responsable de formation FO, face aux prévisibles « va-et-vient (croissants) entre carrières syndicales et carrières professionnelles », entraînés par la loi sur la représentativité de 2008, évoque « une priorité à la formation professionnelle pour les permanents syndicaux » (Journée sur la formation syndicale, ISST Paris 1, 25 juin 2010).

26. SimonPoli (2006), « La VAE du militant », Encadrement magazine, n 139, p. 10.

27. Lors du lancement de l'observation, en 2006 (cf. Encadrement magazine, 2007, n ${ }^{\circ}$ 147, pp. 14-15), un projet analogue est initié par la FCE-CFDT (fédération chimie énergie) : le SVPm (Suivi et valorisation du parcours militant), qui repose sur une série d'entretiens entre le militant en demande de suivi et un « interlocuteur dédié », issu du syndicat et formé à l'exercice.
} 
prud'homale, afin qu'ils puissent par la suite les valoriser en obtenant des équivalences d'unités de valeur de diplômes universitaires. Pour ce faire, la confédération a établi des relations de partenariat avec des universités ${ }^{28}$.

Cette évolution s'accompagne d'une spécialisation et d'une différenciation des niveaux de formation. Le module «Ouverture sur le syndicalisme» ne constitue plus le seul préalable aux stages du CFS : des prérequis sont exigés pour la plupart des formations. Une logique de cursus est de plus mise en œuvre. Par exemple, la formation sur l'Europe, instituée en $1989^{29}$, s'enrichit d'un deuxième stage, sur les comités d'entreprise européens, destiné aux DS et aux délégués syndicaux centraux (DSC) d'entreprises de dimension communautaire et aux membres des CE européens. De même, pour participer au stage « Management de l'équipe syndicale », il faut avoir suivi le module « Développer sa section syndicale».

Accompagnant et reproduisant « la transformation des perceptions de l'engagement que l'on retrouve dans l'ensemble de la vie sociale » dans un sens déconflictualisé, managérial et technicisé (WILLEMEZ, 2006, p. 390), l'introduction de la validation des acquis de l'expérience (VAE) et la rationalisation de l'offre s'inscrivent dans l'affirmation plus large d'un syndicalisme d'adhérents.

\section{Un syndicalisme d'adhérents}

Formation et information font partie des outils permettant le développement syndical. Attractives, présentées et perçues comme un « service » offert aux adhérents mais aussi à des salariés non syndiqués vis-à-vis desquels elles constituent autant de produits d'appel, ces formations laissent une grande place au développement personnel.

\section{Une politique de syndicalisation}

La formation s'inscrit de fait dans une politique mettant l'accent sur les services offerts à l'adhérent ${ }^{30}$, après que le syndicat a un temps proposé, par un « contrat d'engagement social », de réserver aux syndiqués le bénéfice des dispositions des conventions collectives ${ }^{31}$. Lors du $34^{\mathrm{e}}$ congrès national de la CFE-CGC, en 2010,

28. Une convention de partenariat est ainsi signée dès 2010 avec Sciences Po Aix-en-Provence, à l'initiative du Syndicat métallurgie Provence Côte d'Azur-CFE-CGC, pour permettre aux militants ayant suivi des formations syndicales d'accéder à une formation de « correspondant en intelligence sociale ».

29. C'est cette année-là que les principaux syndicats de cadres européens, dont la CFE-CGC, fondent la Confédération européenne des cadres (CEC).

30. Ces services comprennent l'assistance et la protection juridique dans le cadre de la vie professionnelle et syndicale, l'aide aux démarches au sein des institutions et organismes sociaux, telles que l'action logement, ex-1\% logement, la mutuelle santé réservée aux adhérents et à leur famille, l'accompagnement psychologique proposé dans les situations de détresse, etc.

31. CFE-CGC, Manifeste pour un syndicalisme d'avenir et un avenir dans le syndicalisme, Paris, 31 mai 2007 , p. 14. L'hypothèse d'avantages conventionnels réservés aux adhérents avait également été explorée, dans les années 1990 , par la CFDT (Guillaume, Pochic, 2009b). 
l'équipe confédérale confie par ailleurs au secrétaire national de la formation initiale et du dialogue social la mission de développer des actions visant les étudiants et les jeunes diplômés : consolidation des services d'informations et de stages, animation du réseau des représentants CFE-CGC dans les conseils d'administration des établissements d'enseignement supérieur, installation d' « ambassadeurs CFE-CGC » dans les écoles, amélioration du partenariat avec Companieros, organisme de formation au «management responsable » ${ }^{32}$, etc. «Guidée par deux priorités : la VAE syndicale et l'enquête sur les attentes des étudiants vis-à-vis du syndicalisme ${ }^{33} »$, cette action pédagogique et de communication valorise la formation syndicale « au service des adhérents ». La formation est plus largement mise en avant dans le programme de la nouvelle équipe à la tête de la CFE-CGC, qui propose en 2013 un élargissement de l'offre avec « une capacité d'accueil renforcé », d'autant qu' « il y a des subventions que nous ne consommons pas ; donc il est urgent de mettre en place un certain nombre de formations ${ }^{34} »$. Insuffisamment utilisée ${ }^{35}$, elle fait l'objet d'une communication renforcée ces dernières années : le calendrier présentant l'offre de formation est mis en ligne et les annonces sont régulièrement publiées. Les stages sont de plus ouverts, sur dérogation, à des salariés non syndiqués qui, d'après une responsable de formation, « attendent la formation pour prendre leur adhésion ».

Bien que les circonstances et les raisons de l'adhésion soient diverses (volonté d'obtenir de l'information, sollicitation d'un collègue, prise de mandat), les formations sont souvent évoquées par les stagiaires comme une contrepartie attendue de la prise de carte. Par exemple, un cadre, responsable de formation professionnelle dans une entreprise de services, DS et membre du CHSCT, adhérent à la CFE-CGC depuis cinq ans, explique s'être d'abord présenté sans étiquette aux élections du personnel et avoir été élu DP et membre du CE pour s'occuper d'un projet de fusion en cours dans son entreprise ; remarquant que sa position d'élu ne lui permettait malgré tout pas d'avoir toutes les informations nécessaires sur ce projet, il décide finalement de se syndiquer. Il précise qu'il reste syndiqué pour, entre autres raisons, les formations : «En étant syndiqué, j'ai eu accès à des formations, et c'est là que ça devenait intéressant. » Une

\footnotetext{
32. Fondé en 2000 par un dirigeant d'entreprise, cet organisme propose des formations normalisées (par un accord de l'Association française de normalisation [Afnor]) ; des certificats individuels ou « labels » sont décernés sur l'une des thématiques de la diversité en entreprise (Handimanagement, HF management, Pluriel management, etc.) au terme d'une épreuve finale en ligne. Les «équipiers Companieros », intégrés à un réseau d'étudiants labellisés, entament ensuite une campagne de communication sur leur campus. Depuis 2015, une « association des transformeurs » réunit l'ensemble des « labellisés », c'est-à-dire toutes les personnes ayant obtenu un tel label afin d'agir au sein des entreprises et sur les campus.

33. «Représentativité, dialogue social, formation initiale », Encadrement magazine, $\mathrm{n}^{\circ}$ 171, 2010.

34. $35^{\mathrm{e}}$ congrès national de la CFE-CGC, Saint Malo, 17-19 avril 2013, réponses de l'équipe de Carole Couvert : https://www.youtube.com/watch?v=PiltG5ukmlY ; vidéo mise en ligne le 22 mai 2013, consultée le 10 mars 2016. 35. La formation à la CFE-CGC, c'est 6000 journées stagiaires annuelles ; plus de deux adhérents sur trois n'en ont pas suivi en 2010. Les cadres masculins de 36 à 60 ans en constituent la cible principale : cf. Junior Entreprise ESCP-EAP Conseil (2010), Rapport de synthèse pour la CFE-CGC sur la formation syndicale. Ce rapport, commandé par la direction des services confédéraux, insiste sur l'insuffisante information sur l'offre de formation, la nécessité de « jouer sur la formation pour accroître les adhésions à la CFE-CGC » et d' « inclure la formation dans le discours de "vente" du syndicat » (p. 9).
} 
cadre du secteur de l'énergie, syndiquée depuis quatre ans, alors qu'elle était déjà élue $\mathrm{DP}$ et trésorière de son $\mathrm{CE}$, en cours de second mandat, précise même avoir adhéré pour en bénéficier : «C'est pour ça que j’ai adhéré, c'est clair. »

Produit d'appel ou de fidélisation, la formation est également mobilisée comme outil de développement des sections. Dès la formation «Ouverture sur le syndicalisme », l'adhérent est placé au centre de l'organisation (voir encadré 5).

\section{ENCADRÉ 5}

\section{L'adhérent, au centre de l'organisation}

Le formateur introduit le stage « Ouverture sur le syndicalisme » par la « Question des questions : au centre de tout le système, c'est qui ?» Un stagiaire s'écrie : «BVC ! [Bernard Van Craeynest] ». Suite au signe de dénégation du formateur, un autre avance : «Les adhérents ? » Immédiatement, le formateur lance l'image de son diaporama Powerpoint montrant des adhérents avec, pour fond sonore, des applaudissements, avant d'opiner : « Sans les adhérents : vous n'avez pas de cotisation ! Même BVC est adhérent ! » Il précise ensuite : « La confédération s'occupe de tous les salariés. » Suivent le trombinoscope de la confédération et une présentation des fonctions respectives des responsables ; le formateur distingue les militants des salariés « experts » de la confédération, dont il fait partie, et le militant de l'adhérent : «L'adhérent est celui qui cotise. » Le militant « exerce des mandats, s'investit, a pour rôle de "faire des adhérents", "communiquer", "informer" ».

Notes d'observation du stage « Ouverture sur le syndicalisme », janvier 2008.

Sur fond de désyndicalisation et de mise en concurrence croissante de représentants qui doivent convaincre pour être (ré)élus suite à la réforme de la représentativité de 2008, la communication est devenue un enjeu ${ }^{36}$. Elle figure en bonne place dans l'argumentaire des stages ${ }^{37}:$ "Créer et développer sa section syndicale » vise par exemple à étendre une «culture de l'adhésion » dans les sections syndicales ${ }^{38}$. L'animateur, juriste salarié de la confédération, souligne qu'il faut « donner envie aux gens de vous rejoindre dans la section ». Cherchant à « équiper pour agir » mais aussi à développer

\footnotetext{
36. Sur la promotion d'une politique de communication et de « développeurs » à la CFDT, où la formation constitue un pivot pour convaincre les militants et les outiller, cf. Guillaume, Pochic, 2009b. Sur la communication, enjeu de formation aux contours incertains pour les syndicats et les ISST, cf. OLIVESI, 2011.

37. Un stage, récent, de « communication écrite » évoque la stratégie de communication et les écueils à éviter (« ce que vous devez et ne devez surtout pas faire ») : Encadrement magazine, $\mathrm{n}^{\circ} 202,2014$, p. 7.

38. Pour un parallèle avec la CFDT, voir Gulllaume, Pochic, 2009b. Pour espérer syndiquer leurs collègues, il faut que les militants disposent d'un solide outillage cognitif. L'enjeu est de taille; des formations spécifiques sont ainsi mises en place afin de leur permettre de répondre aux demandes des salariés lors d'une première prise de contact. Une formation sur le contrat de travail est, par exemple, proposée, car, souligne une responsable de formation, « quand ils [les militants] sont interrogés, approchés par leurs collègues de l'entreprise, très souvent, la première fois, c'est par rapport à leur contrat $[\ldots]$ ou celui de leur conjoint ».
} 
le leadership, la formation a pour dessein à la fois l'empowerment $t^{39}$ individuel et le développement syndical. Dans la lignée de l'objectif de « revitalisation » du mouvement syndical et à l'instar des méthodes d'organizing (Voss, SHERMAn, 2000) mises en œuvre par ses homologues anglo-saxonnes ou germaniques, la confédération met en avant la proximité, l'écoute et mobilise la formation pour favoriser la prise de carte.

L'insistance sur la nécessité de recruter de nouveaux adhérents suscite toutefois des résistances chez des stagiaires qui, confrontés au renouvellement de leurs équipes, ne la mentionnent pas comme une mission prioritaire. Ils sont avant tout soucieux de l'investissement des syndiqués. Ainsi, une stagiaire, cadre du secteur bancaire, cumulant les responsabilités, est plutôt tentée de demander au nouvel adhérent : «Qu'est-ce que tu m'apportes ? L'enjeu est en effet, selon une permanente syndicale « de transformer des adhérents en militants, [...] de pas tourner toujours avec les mêmes $[\ldots]$; on n'est pas des professionnels du syndicalisme ». Les stagiaires que nous avons rencontrés mettent de fait principalement en avant le décalage entre leur investissement et celui des membres de leur section.

\section{Le développement personnel}

Les formations sont particulièrement appréciées en tant que moments propices aux échanges d'expérience, qui permettent de relativiser les obstacles rencontrés et de sortir d'une approche individualisante des difficultés. Elles concourent à une forme de « réparation psychologique », telle que celles analysées par Hélène STEVENS (2013) dans le cadre des formations en « développement personnel ». Ainsi, un élu CHSCT, fonctionnaire dans une entreprise de télécommunications, souligne : «S'il y a eu des fois où j'étais pas bien, où j'ai pris des coups,... chaque fois j'essayais d'aller en formation pour me rebooster. »

Le stage « Expression vocale et gestuelle » est explicitement orienté vers le développement personnel. Selon une responsable de formation, «l'animateur leur [aux stagiaires] fait du bien, parce qu'il ne parle pas de syndicalisme. [...] Ils en tirent le fait de passer deux jours en confiance, avec quelqu'un qui les met en valeur, ça leur fait un bien considérable. C'est aussi ça, sinon, ils ne gèrent que de la misère, que des choses difficiles ».

Les motivations qu'ont les participants pour ce type de stage sont diverses : « apprendre à parler », « à respirer, à poser [sa voix] », à mieux maîtriser ses émotions et son «stress ». Selon un élu CHSCT, il s'agit de renforcer la « confiance en soi », d'avoir davantage de « réflexivité » et de capacité de mise à distance, notamment pour moins « monter au créneau », insiste un médecin du travail, DP et membre de CE.

Les stagiaires sont, pour certains, sensibilisés à ces approches par leurs expériences personnelles, leurs activités ou projets professionnels. Par exemple, un DS,

39. C'est-à-dire une « mise en capacité », une « capacitation », au sens d'accroissement du pouvoir d'agir des individus sur les conditions sociales, économiques, politiques ou écologiques qu'ils subissent. 
DP et élu CHSCT participant à l'Observatoire du stress et des mobilités forcées à France Télécom, se passionne pour le « développement personnel » et souhaite créer une entreprise dans ce domaine.

Cette préoccupation, qui va de pair avec le souci croissant de former au management et à la négociation (cf. infra), concerne aujourd'hui l'ensemble des formations destinées aux cadres, y compris celles d'autres confédérations, telle la CFDT (Guillaume, 2011), qui tendent ainsi à se rapprocher des stages mis en place par la CFE-CGC depuis plus de trente ans (DELmas, 2011).

\section{La promotion de la figure du manager-négociateur}

L'essor de l'offre de formations en management et en négociation s'accompagne d'un recours croissant à des techniques managériales ; il invite à interroger ce que « savoir négocier» veut dire à la CFE-CGC.

\section{L'utilisation de techniques managériales et de coaching}

La promotion de la figure du manager se concrétise à la CFE-CGC par les formations « Management de l'équipe syndicale », « Développer sa section syndicale », « Négociation, niveaux 1 et 2 », auxquelles s'ajoute l'expérimentation de sessions consacrées aux "négociations difficiles », aux interactions conflictuelles, ou aux «sujets qui fâchent $»^{40}$.

Lors des formations axées sur l'apprentissage des rôles et prérogatives du manager syndical, nous avons pu constater de nombreux glissements entre le registre syndical et le registre professionnel. C'est d'autant plus le cas qu'elles sont destinées à des stagiaires exerçant professionnellement des fonctions managériales. Le prestataire animant le stage " Management de l'équipe syndicale » pointe ainsi qu' « un système ne fonctionne pas sans leader », et souligne la spécificité d'une parole à destination « des cadres, souvent des managers ». Le manager hiérarchique étant aujourd'hui essentiellement fonctionnel et de moins en moins décisionnel, il s'agit sur le plan méthodologique de travailler « de plus en plus par la notion de délégation et de représentation ». Les difficultés liées au management sont mises en avant par les stagiaires. «Qu'est-ce qu'on entend par management ? interroge un DS et DP dans une grande entreprise de transport urbain : « Il existe une ambiance mais il n'y a pas réellement de management. Quand on donne des responsabilités, on a du mal à se faire entendre. »

Pour faire face à ces difficultés, plusieurs techniques sont proposées, dont la promotion s'inscrit dans une nouvelle culture psychologique mettant l'accent sur les capacités et l'autonomie humaines. En lien avec l'essor d'un néo-management,

40. CFS, Calendrier des sessions interprofessionnelles, $2^{\mathrm{e}}$ session 2015, juin 2015, p. 6. Cette formation apparaît dans le calendrier des sessions interprofessionnelles en 2015. 
proposition est faite à chacun de se développer personnellement, via la mobilisation de coachs (BolTANSKi, ChiAPPELlo, 1999) et un travail sur la subjectivité devenant sa propre fin par le recours aux psychotechnologies (pour une évocation ancienne, CASTEL, 1981).

La mobilisation de méthodes managériales et de coaching présente ici plusieurs spécificités : elles tiennent à leur ancienneté, si l'on se réfère à la mise en place par la FNSIC, au début des années 1970, de formations psychosociologiques orientées vers la communication et l'approfondissement du mode d'être en groupe (DELMAS, 2011) ; elles renvoient également aux techniques utilisées, l'analyse transactionnelle reposant par exemple sur le postulat d' «états du moi » actualisés lors d'interactions interpersonnelles, le Myers Briggs Type Indicator (MBTI) déterminant le «type psychologique » d'un sujet à partir de «dominantes »; enfin, elles sont singulières, parce qu'externalisées à des formateurs œuvrant auprès de publics cadres pour des entreprises et des organisations patronales.

Ces formateurs mettent en avant leur activité de coach et de consultants spécialisés « dans l'accompagnement au changement des dirigeants et cadres » auprès de grandes entreprises. L'un d'entre eux met aussi l'accent sur ses activités de psychothérapeute et de psychanalyste. Un autre, devenu huit ans auparavant consultant indépendant après avoir travaillé un an dans le secteur des transports, insiste sur sa double activité, de « conseil » et de coaching où il s'agit d' « aider les gens à réfléchir ». Autant de figures qui rappellent les trajectoires des coachs décrits par Scarlett SALMAN (2015).

Comme les formations professionnelles en management proposées par les entreprises (COUSIN, 2008), ces stages sont centrés sur le comportement, les « technologies de soi » et l' « action sur soi » (ERALY, 1994). Les vertus de techniques relationnelles avec ses subordonnés, ses chefs, ses collaborateurs, voire son conjoint et ses enfants, sont valorisées. Il s'agit de mieux se connaître pour accroître l'efficacité de sa relation à autrui dans le cadre de démarches présentées comme résolument " pragmatiques ». À l'instar du coaching (SALMAN, 2008) ou de l'accompagnement, les relations sont vues dans leur dimension psychologique, le postulat étant que la communication, le « développement personnel » et l'efficacité syndicale sont étroitement imbriqués. Ces techniques, fournies par des «managers de l'âme » (BRUNEL, 2004), proposent des exercices pratiques de transformation de soi devant aider les militants à interagir avec autrui et à conduire les autres en renforçant leur «potentiel », leur « confiance en eux-mêmes », leur « estime de soi ».

L'animateur de la formation «Management de l'équipe syndicale » insiste d'ailleurs particulièrement sur l'importance des relations interpersonnelles, le « rôle charismatique » du délégué syndical et les particularités du «management » de la section qui, dénué de dimension hiérarchique, est un «mode de management transversal ou fonctionnel ou coopératif », parfois moins simple qu'en entreprise, impliquant d'être un « délégué coopérant », c'est-à-dire, « être dynamique, avoir une énergie communicative ». 
La réceptivité des stagiaires à cette vision et à ces techniques est sans doute facilitée par leur profil et leurs fonctions (commerciales, managériales, logistiques ou de gestion). La « bascule de génération » est évoquée par un secrétaire CE et DS dans une entreprise informatique, comme impliquant une culture plus coopérative, par opposition à la précédente génération « qui avait le culte du chef ». Si la valeur hiérarchique est toujours essentielle dans l'identité cégéciste (BÉTHOUX et al., 2013), le renouvellement générationnel et la féminisation des adhérents et de l'encadrement syndical, mais aussi la nécessité, désormais, de prouver son audience avec la réforme de la représentativité, contribueraient à la promotion de cette figure précitée du « délégué coopérant». L'objectif peut être, selon un DS d'une entreprise de distribution ayant essuyé un échec électoral, de « se donner les moyens de se recrédibiliser vis-à-vis des salariés qui nous ont tourné le dos ».

Les attentes à l'égard du stage sont toutefois contrastées ; s'il s'agit pour l'un des participants de contribuer à « consolider » le développement de sa section, la plupart sont plutôt confrontés au renouvellement de leur équipe et au manque de motivation des adhérents ; une stagiaire DS du secteur de la banque et des assurances, proche de la retraite et souhaitant préparer sa succession, souligne ainsi la démotivation de son équipe, jeune et en pleine mobilité professionnelle, dans un contexte de mise en minorité syndicale. Plusieurs stagiaires souhaitent « déléguer plus » et « développer un esprit d'équipe ». Un participant cumulant plusieurs mandats, est en attente d'échanges sur « comment organiser des missions au sein de l'équipe avec des caractères et des engagements différents », «faire monter en compétence » et « donner envie de s'investir plus pour que l'on puisse déléguer un peu plus ».

L'essor du champ conventionnel et l'enfermement des syndicalistes dans des activités chronophages telles que la conduite de réunions et la préparation de dossiers (GIRAUD, 2013) renforcent ce souhait et celui de se familiariser à la négociation.

\section{L'apprentissage de la négociation}

La formation à la négociation prend une importance cruciale aujourd'hui pour des syndicats confrontés à l'intensification du rythme des négociations d'entreprise. L'ouverture, par la CFE-CGC, de stages consacrés à cette question, loin d'être une singularité, s'inscrit dans un mouvement plus vaste. Toutefois, son intérêt est particulièrement souligné au sein d'une confédération où les pratiques militantes sont fortement institutionnalisées (comme en témoigne l'importance des mandats) et où le compromis est privilégié (BÉTHOuX et al., 2013) à l'instar de la CFDT (GUILLAUME, PoCHIC, 2009a). Les nouvelles règles du paritarisme et les enjeux de la formation des élus des instances paritaires, évoqués lors du $35^{\mathrm{e}}$ congrès national de la Confédération ${ }^{41}$, renforcent encore les attentes à l'égard de ces formations.

41. $35^{\mathrm{e}}$ congrès national de la CFE-CGC, Saint Malo, 17-19 avril 2013, réponses de l'équipe de Carole Couvert : https://www.youtube.com/watch?v=Pi1tG5ukmlY ; consulté le 18 mars 2016. 
Elles peuvent être de différents types. Certaines sont centrées sur des sujets de négociation. Ainsi, « Négocier un accord de QVT » est inscrit au calendrier, en lien avec l'accord national interprofessionnel du 19 juin 2013 et, plus spécifiquement, le rôle du personnel d'encadrement sur cette question complexe et le positionnement précoce de la CFE-CGC sur le « stress » des cadres (DELMAS, 2010). L'épargne salariale fait également l'objet d'une formation, conformément à une volonté de diffusion des positionnements confédéraux sur cette question. D'autres formations portent, plus généralement, sur les « techniques de négociation ». À l'instar des autres formations, les statuts professionnels et les niveaux de qualification des stagiaires y sont contrastés, comme leurs mandats, qui ne se limitent pas à celui de délégué syndical.

Les attentes des stagiaires envers ces formations « techniques » rendent compte d'un enjeu de crédibilisation face à la direction, dans un contexte de durcissement des négociations. Ainsi, une militante, commerciale dans une société d'assurances en fin de carrière, adhérente depuis onze ans, et fortement investie syndicalement à la suite de fusions et de réorganisations incessantes dans son entreprise, indique : « En face de nous on a vu arriver de plus en plus des professionnels [...], des machines à négocier et nous, on manque un peu de technique, on nous enferme dans des dossiers, dans du travail. [...] Parfois on n'est pas assez pro. » Il s'agit de « connaître les techniques de négociation », « les mots, la technique des mots à utiliser lors des négociations »; « on n'utilise pas toujours les bons mots, les bons termes », souligne un élu CE, syndiqué depuis quatre ans à la fédération métallurgie. Ce contexte difficile implique de se professionnaliser, de « savoir négocier car jusqu'à maintenant je marchais au feeling », « d'autant que maintenant les patrons ne lâchent plus rien », indique un permanent syndical exerçant dans le secteur des télécommunications. Il peut ainsi s'agir d'appréhender « jusqu' où on peut aller dans la négociation »; « on nous dit souvent : "Vous ne demandez pas suffisamment." [...] J'ai testé personnellement mais c'est pas d'une folle évidence », remarque un agent technique dans une fonderie, DS et élu CE. Les objectifs sont de « prendre de l'assurance pendant la négociation et tirer parti de l'expérience de tout le monde », insiste un informaticien, DS, DP et membre de $\mathrm{CE}, \mathrm{d}$ ' " arriver à convaincre, [...] de mettre en avant les arguments et qu'ils soient écoutés, car on a trop l'habitude d'être avec nos collaborateurs », précise un DS, cadre de l'industrie automobile. Pour une DP et DS « novice » qui « n'a jamais participé à des négociations », informaticienne travaillant dans le secteur des assurances, il s'agit de « profiter de l'expérience des sachants, connaître un peu les techniques, les stratégies qui peuvent être appliquées ». Si ce sont les discussions avec la direction qui sont le plus souvent analysées, les formations abordent aussi les échanges avec les autres syndicats et les militants ; deux stagiaires du même syndicat insistent sur la nécessité de parvenir à « se mettre d'accord entre nous avant de négocier » et de « se préparer ». Une DS, cadre commerciale d'une société de protection sociale, mentionne pour sa part le souci d' « avoir une éthique pour ne pas systématiquement être en opposition », dans un contexte où, confrontés à trois autres syndicats, dont la CFDT majoritaire, « nous 
sommes presque à signer tous les accords tout seuls. [...] On est presque obligé de se mettre en opposition pour pouvoir se faire entendre ».

Ces soucis de préparation, professionnalisation et crédibilisation ne sont pas spécifiques aux militants cégécistes ; ils témoignent, toutefois, d'une proximité avec la direction qui est parfois perçue comme encombrante dans le cadre des négociations. Si on peut faire l'hypothèse de définitions syndicalement contrastées de ce que signifie « négocier » (GANTOIS, 2014, p. 293), la CFE-CGC semble se caractériser par la promotion de la figure du « médiateur », à l'instar de la CFDT. L'observation de stages de formation rend de fait compte du souhait d'amener les stagiaires à être aptes au compromis, d'où la mobilisation de savoirs de type relationnel ou managérial. Cette caractéristique rapproche la formation cégéciste de celles proposées par la CFDT et FO, qui ont recours aux savoirs à dimension managériale, la CFDT s'appuyant toutefois davantage sur les sciences sociales et les savoirs académiques (dont la sociologie), FO sur l'économie (GANTOIs, 2014). Moins ouverte aux sciences sociales et moins liée au monde universitaire, la CFE-CGC se caractérise par un recours ancien aux techniques psychosociologiques, comme nous l'avons dit. Les savoirs mobilisés la distinguent de la CGT, où les formations à la négociation s'appuient sur le droit, pensé comme une ressource à articuler avec d'autres modes d'action syndicale (GANTOIS, 2014, p. 298), et rejettent les savoirs à dimension managériale. La délégation des formations à des prestataires de services constitue une autre particularité de la CFECGC, comparativement aux autres organisations syndicales, dont les formateurs, qu'ils soient permanent syndicaux ou non, se caractérisent par une expérience de négociation d'entreprise ou de branche, ou par une connaissance des institutions de négociations (GANTOIS, 2014). Enfin, si les jeux de rôle, très mobilisés dans les stages que nous avons observés, le sont aussi dans ceux proposés par les autres confédérations, ils y attestent d'un brouillage plus évident entre les registres professionnel et syndical. Les « mises en situation » et études de cas sont, en effet, pour la plupart extérieures aux situations syndicales et empruntent aux techniques de négociation commerciale ou de vente d'un produit. Ces jeux de rôle sont d'ailleurs appréciés par des stagiaires amenés à négocier pour des raisons tout autant professionnelles que syndicales. Ainsi, une DP, cadre du secteur des télécommunications, souligne avoir particulièrement été marquée, dans le stage, par « les jeux de rôle [...] pour arriver à convaincre. [...] Moi, ça me sert également dans mon métier de tous les jours... je passe mon temps à négocier... »

On peut s'interroger sur l'assimilation sous-jacente de la négociation à une pratique déconnectée de ses enjeux politiques et de son encadrement juridique (GANTOIS, 2014), négociation légitimée comme une « fin en soi » par une confédération qui se concentre sur l'apprentissage de méthodologies et de savoirs relationnels de type managérial. 
Fruit de l'agrégation de divers systèmes de représentation et de strates socioprofessionnelles composites, la culture organisationnelle de la CFE-CGC repose sur la dénégation des divisions. La volonté d' " asseoir un positionnement rassembleur », d' « élaborer un positionnement clair », de « gagner en légitimité et crédibilité », d' " améliorer sa visibilité et sa notoriété » au service d' " un syndicalisme constructif et responsable ${ }^{42} »$, est également congruente avec le refoulement de la dimension idéologique du syndicalisme et la promotion d'une forme d'humanisme structuré autour de la notion de responsabilité. La compétence et l'expertise sont expressément citées et la formation présentée comme un outil de valorisation et de perfectionnement des compétences professionnelles.

$\mathrm{Si}$, à la CFE-CGC, la formation syndicale est voulue comme essentiellement pratique, et si la rationalisation de ces dernières années renforce cette orientation, elle n'en véhicule pas moins, dans le prolongement de l'histoire de la confédération, des valeurs et des normes militantes. Le dessein de ne pas ériger une frontière entre les registres professionnel et syndical demeure fort, comme en atteste cette réflexion d'une responsable de formation : «Moi, je suis en entreprise, je suis cadre, je suis agent de maîtrise, comment j'intègre cette problématique et comment dans mon activité professionnelle je peux donner un angle... durable, sociétal à mon action quotidienne ? »

S' appuyant sur une forme d'idéologie de la non-idéologie, mobilisant des valeurs professionnelles et entrepreneuriales, la formation syndicale à la CFE-CGC révèle à la fois des évolutions affectant l'ensemble de l'espace syndical et la singularité d'une identité ayant l'entreprise comme espace de référence.

\section{BIBLIOGRAPHIE}

Amossé T. (2004), « Mythes et réalités de la syndicalisation en France », Premières informations, premières synthèses, Paris, Dares, $\mathrm{n}^{\circ}$ 44-2.

Amossé T. (2010), « Cadres/non-cadres : une frontière toujours consistante », in Bouffartigue P., Gadéa C., Pochic S. (dir.), Cadres, classes moyennes, vers l'éclatement?, Paris, Armand Colin, pp. 32-45.

Amossé T., Delteil V. (2004), «L'identité professionnelle des cadres en question », Travail et Emploi, n ${ }^{\circ}$ 99, pp. 63-79.

Bensoussan M. (2010), L'Engagement des cadres. Pratiques collectives et offres de représentation, Paris, L'Harmattan.

BÉROUd S., YON K. (coord.) (2011), La Loi du 20 août 2008 et ses implications sur les pratiques syndicales en entreprise : sociologie des appropriations pratiques d'un nouveau dispositif juridique, rapport de recherche, convention Dares «Impact des nouvelles règles de la représentativité sur les pratiques et les stratégies syndicales ».

42. La Lettre confédérale, $\mathrm{n}^{\circ} 1271,10$ octobre 2011. 
Béthoux É., Desage G., Mias A., Pélisse J. (2011), « La "drôle de crise" de la CFE-CGC : hésitations stratégiques et distorsions organisationnelles d'un syndicalisme catégoriel », Travail et Emploi, n ${ }^{\circ} 128$, pp. 5-22.

Béthoux É., Desage G., Mias A., Pélisse J. (2013), Sociologie d'un syndicalisme catégoriel. La CFE-CGC ou la fin d'une exception?, Paris, Armand Colin.

Boltanski L. (1982), Les Cadres : la formation d'un groupe social, Paris, Éditions de Minuit. Boltanski L., Chiapello È. (1999), Le Nouvel Esprit du capitalisme, Paris, Gallimard.

Bouffartigue P. (2001), «Les métamorphoses d'un salariat de confiance : les cadres dans le tournant des années quatre-vingt-dix », Travail et Emploi, $\mathrm{n}^{\circ}$ 86, pp. 107-125.

Bouffartigue P., Gadéa C., Pochic S. (dir.) (2010), Cadres, classes moyennes, vers l'éclatement?, Paris, Armand Colin.

BRUNEL V. (2004), Les Managers de l'âme : le développement personnel en entreprise, nouvelle pratique de pouvoir?, Paris, La Découverte.

CADET J.-P., MöBUs M. (2011), La Promotion au statut de cadre des professions intermédiaires. Analyse quantitative, enquêtes en entreprise, Recherche en partenariat Céreq/Apec, Paris, Apec.

CAmbus C. (2003), « La formation syndicale à la CFE-CGC », Éducation permanente, $\mathrm{n}^{\circ} 154$, pp. 71-79.

CASTEl R. (1981), La Gestion des risques : de l'anti-psychiatrie à l'après-psychanalyse, Paris, Éditions de Minuit.

CAstelot A.-S. (2004), « Le foyer syndical, au cœur d'une pratique syndicale », Cahiers du GDR cadres, $\mathrm{n}^{\circ}$, pp. 147-153.

Cornu T., Groux G. (2006), « Un cadrage historique. Sources et ressources des mobilisations collectives des cadres. Du "rebelle" collectif à l'individu "rebelle" ? », Cahiers du GDR cadres, $\mathrm{n}^{\circ} 12$, pp. 6-16.

Cousin O. (2008), Les Cadres à l'épreuve du travail, Rennes, Presses universitaires de Rennes.

Delmas C. (2010), « Le stress des cadres : enjeux syndicaux et médiatiques », in Bouffartigue P., Gadéa C., Pochic S. (dir.), Cadres, classes moyennes, vers l'éclatement ?, Paris, Armand Colin, pp. 249-259.

Delmas C. (2011), « Du "perfectionnement" professionnel à la "performance" de l'action syndicale. Formations et expertise à la Confédération française de l'encadrement-Confédération générale des cadres », Le Mouvement social, n 235, pp. 89-104.

ERALY A. (1994), «L'usage de la psychologie dans le management : l'inflation de la "réflexivité professionnelle" », in Bouilloud J.-P., Lécuyer B.-P. (dir.), L'Invention de la gestion. Histoire et pratiques, Paris, L'Harmattan, pp. 135-159.

Ethuin N., Siblot Y. (2011), « Du cursus d'éducation syndicale aux parcours de formation des militants de la CGT (années 1950-2000) », Le Mouvement social, n ${ }^{\circ}$ 235, pp. 53-69.

Ethuin N., Yon K. (2010), « Entre travail, citoyenneté et militantisme : un panorama des travaux sur les relations polyphoniques entre syndicalisme et formation », Savoirs, n ${ }^{\circ}$ 24, pp. 9-57.

GANTOIS M. (2014), «Former à la négociation collective. Observations croisées de stages à la CFDT, à la CGT et à FO », in Ethuin N., Yon K. (dir.), La Fabrique du sens syndical. 
La formation des représentants des salariés en France, 1945-2010, Bellecombe-en-Bauges, Éditions du Croquant, pp. 291-313.

GiRAUD B. (2013), « Derrière la vitrine du "dialogue social” : les techniques managériales de domestication des conflits du travail », Agone, n ${ }^{\circ} 50$, pp. 33-63.

Groux G. (1983), «Les couches moyennes, l'État et le corporatisme en France », Revue française de sociologie, vol. 24, $\mathrm{n}^{\mathrm{o}} 2$, pp. 301-315.

Groux G. (1990), « Le syndicalisme-cadres de l'après-guerre à nos jours. Légitimation sociale et "affirmation de soi” », in Reynaud J.-D., Eyraud F., Paradeise C., Saglio J. (dir.), Les Systèmes de relations professionnelles. Examen critique d'une théorie, Paris, CNRS Éditions, pp. 133-145.

Grunberg G., Mouriaux R. (1979), L'Univers politique et syndical des cadres, Paris, Presses de la Fondation nationale des sciences politiques.

Guichet C. (2015), « Les forces vives au féminin », Les Études du Conseil économique, social et environnemental, $\mathrm{n}^{\mathrm{o}} 32$.

GuILlaume C. (2011), « La formation des responsables à la CFTC-CFDT : de la "promotion collective" à la sécurisation des parcours militants (1950-2010) », Le Mouvement social, $\mathrm{n}^{\circ}$ 235, pp. 105-119.

Guillaume C., Pochic S. (2009a), « Un engagement incongru ? Les cadres et le syndicalisme, l'exemple de la CFDT », Revue française de science politique, vol. 59, $\mathrm{n}^{\circ} 3, \mathrm{pp} .535-568$.

Guillaume C., Pochic S. (2009b), «La professionnalisation de l'activité syndicale : talon d'Achille de la politique de syndicalisation à la CFDT ? », Politix, $\mathrm{n}^{\circ}$ 85, pp. 31-56.

HENRY O. (intro.) (2012), «Expertise, syndicalisme et conditions de travail », Revue de l'Ires, $\mathrm{n}^{\mathrm{o}} 74$, pp. 63-206.

LESTIENNE B. (1979), Perspectives politiques et idéologiques de la formation syndicale à la CFDT, thèse d'économie, Paris 1.

Lombardo M. (2005), Formation et pédagogie syndicales CGT et CFDT 1968-1985 : en relation au droit social, à l'évolution de la société, aux mutations du monde du travail, thèse d'histoire, Paris 1.

MEYER M. (1978), La Formation des militants dans les syndicats et partis de gauche. L'exemple de la CFDT, thèse de doctorat de $3^{\mathrm{e}}$ cycle en sociologie, Paris 8.

Mouriaux R. (2013), Le Syndicalisme en France depuis 1945, 4 éd., Paris, La Découverte.

NiCOURD S. (dir.) (2009), Le Travail militant, Rennes, Presses universitaires de Rennes.

OLIVESI S. (2011), « La formation en communication des responsables syndicaux. Logiques institutionnelles et enjeux militants », Réseaux, $\mathrm{n}^{\circ}$ 170, pp. 163-189.

OlivieR M.-H. (2006), « Au cœur des mutations du Xx ${ }^{\mathrm{e}}$ siècle : le fonds Roger Millot », Journal de la BDIC, $\mathrm{n}^{\circ} 14$, pp. 4-5.

Pernot J.-M. (2005), Syndicats : lendemains de crise ?, Paris, Gallimard.

Pernot J.-M, Pignoni M.-T. (2008), « Les salariés et les organisations syndicales de 1992 à 2004 : une longue saison de désamour », in Amossé T., Bloch-London C., Wolff L. (dir.), Les Relations sociales en entreprise. Un portrait à partir des enquêtes Relations professionnelles et 
négociations d'entreprise, REPONSE 1992-1993, 1998-1999, 2004-2005, Paris, La Découverte, pp. 140-162.

SALMAN S. (2008), « La fonction palliative du coaching en entreprise », Sociologies pratiques, $\mathrm{n}^{\mathrm{o}} 17$, pp. 43-54.

SALMAN S. (2015), « Le temps des coachs ? Trajectoires typique d'une figure du "nouvel esprit du capitalisme" », Travail et Emploi, $\mathrm{n}^{\circ} 143$, pp. 59-73.

SAWicki F., SiméAnt J. (2009), « Décloisonner la sociologie de l'engagement militant. Note critique sur quelques tendances récentes des travaux français », Sociologie du travail, vol. 51, $\mathrm{n}^{\mathrm{o}} 1$, pp. 97-125.

SiLVERA R. (2006), «Le défi de l'approche intégrée de l'égalité pour le syndicalisme en Europe », Revue de l'Ires, $\mathrm{n}^{\circ}$ 50, pp. 137-172.

SIMÉANT J. (2009), « Socialisation catholique et biens de salut dans quatre ONG humanitaires françaises », Le Mouvement social, n 227, pp. 101-122.

STEVENS H. (2013), « Entre émancipation symbolique et reproduction sociale. Ethnographie d'une formation de "développement personnel” », Travail et Emploi, n ${ }^{\circ} 133$, pp. 39-51.

TomÀs J.-L., KloEtZer L., Clot Y. (2014), « Coanalyser l'activité syndicale : un référentiel d'activité entre dilemmes et acquis de l'expérience syndicale », Nouvelle Revue de psychosociologie, $\mathrm{n}^{\mathrm{o}} 18$, pp. 177-194.

Voss K., SHERMAN R. (2000), "Breaking the Iron Law of Oligarchy: Union Revitalization in the American Labor Movement", American Journal of Sociology, vol. 106, n 2, pp. 303-349.

WiLLEMEZ L. (2003), « Quand les syndicats se saisissent du droit. Invention et redéfinition d'un rôle », Sociétés contemporaines, $\mathrm{n}^{\circ}$ 52, pp. 17-38.

WiLLEMEZ L. (2006), « Faire fructifier son engagement : conséquences et limites de la validation des expériences militantes », in Neyrat F. (dir.) (2006), La Validation des acquis de l'expérience. La reconnaissance d'un nouveau droit, Bellecombe-en-Bauges, Éditions du Croquant, pp. 377-394.

Yon K. (2009), « Quand le syndicalisme s'éprouve hors du lieu de travail. La production du sens confédéral à Force ouvrière », Politix, nº 85, pp. 57-79. 


\section{AnNeXeS}

TABLEAU 1 - Sexe et mandats des stagiaires, par type de formation

\begin{tabular}{|c|c|c|c|c|c|c|c|c|c|}
\hline \multirow[b]{3}{*}{ Formations } & \multirow{3}{*}{ Effectifs } & \multicolumn{2}{|c|}{ Femmes } & \multicolumn{6}{|c|}{ Mandats } \\
\hline & & \multirow[b]{2}{*}{ (Effectif) } & \multirow[b]{2}{*}{$(\%)$} & \multirow{2}{*}{$\begin{array}{c}\text { Nombre } \\
\text { moyen }\end{array}$} & DS & $\mathbf{C E}$ & DP & CHSCT & Autres* \\
\hline & & & & & \multicolumn{5}{|c|}{$(\%)$} \\
\hline Ouverture & 17 & 6 & 35 & 1,4 & 53 & 25 & 71 & 6 & 6 \\
\hline Représentants du personnel & 19 & 11 & 58 & 1,5 & 42 & 22 & 58 & 16 & 16 \\
\hline Élections professionnelles & 9 & 1 & & 4,1 & 100 & 56 & 55 & 33 & 57 \\
\hline Expression vocale et gestuelle & 11 & 4 & 36 & 2,5 & 64 & 46 & 55 & 36 & 27 \\
\hline Argumentation & 15 & 4 & 27 & 2,3 & 60 & 47 & 40 & 53 & 13 \\
\hline Négociation I & 15 & 5 & 33 & 2,5 & 100 & 48 & 47 & 20 & 40 \\
\hline Comité d'entreprise & 17 & 10 & 59 & 1,9 & 29 & 53 & 35 & 18 & 59 \\
\hline Management de l'équipe syndicale & 16 & 5 & 31 & 3,1 & 100 & 56 & 50 & 19 & 81 \\
\hline Négociation II & 13 & 4 & 31 & 2,2 & 100 & 62 & 39 & 15 & 23 \\
\hline Ensemble & $132^{* *}$ & 50 & 38 & 2,0 & 72 & 47 & 48 & 23 & 35 \\
\hline
\end{tabular}

* Délégué syndical central, conseiller du salarié, président de syndicat, secrétaire d'union départementale, responsable syndical, responsable syndical de CE, trésorier de CE, administrateur, conseiller prud'homme, etc.

** 132 réponses, 117 stagiaires au total, en comptant les multiples inscriptions.

Sources : questionnaires et fiches « Restons en contact» (neuf formations).

TABLEAU 2 - Niveau de diplôme des stagiaires, par type de formation

\begin{tabular}{|c|c|c|c|c|c|c|c|c|c|c|}
\hline \multirow{3}{*}{$\frac{\text { Stage }}{\text { Ouverture }}$} & \multirow{3}{*}{$\begin{array}{c}\text { Effectifs } \\
15\end{array}$} & \multicolumn{9}{|c|}{ Niveau de diplôme } \\
\hline & & \multicolumn{2}{|c|}{$\begin{array}{c}\text { Niveau V } \\
(\text { Effectif) }(\%)\end{array}$} & \multicolumn{2}{|c|}{$\begin{array}{r}\text { Niveau IV } \\
(\text { Effectif) }(\%)\end{array}$} & \multicolumn{2}{|c|}{$\begin{array}{r}\text { Niveau III } \\
(\text { Effectif) }(\%)\end{array}$} & \multirow{2}{*}{$\begin{array}{c}\begin{array}{c}\text { Niveau II } \\
\text { (Effectif) }\end{array} \\
1\end{array}$} & \multicolumn{2}{|c|}{$\begin{array}{r}\text { Niveau I } \\
\text { (Effectif) (\%) }\end{array}$} \\
\hline & & & & 4 & 27 & 3 & 18 & & 7 & 47 \\
\hline Représentants du personnel & 15 & & & 3 & & 7 & 47 & 2 & 3 & \\
\hline Élections professionnelles & 7 & 2 & 29 & 2 & 29 & 2 & 29 & & 1 & \\
\hline Expression vocale et gestuelle & 10 & & & 1 & & 4 & 40 & 2 & 3 & 30 \\
\hline Argumentation & 14 & & & 2 & & 7 & 50 & 2 & 3 & 21 \\
\hline Ensemble & $61^{*}$ & 2 & & 12 & & 23 & 38 & 7 & 17 & 28 \\
\hline
\end{tabular}

* 71 stagiaires, 10 non-réponses.

Source : questionnaires (cinq formations).

TABLEAU 3 - Âge et ancienneté syndicale (en années) des stagiaires, par type de formation

\begin{tabular}{lccccc}
\hline Formations & Effectifs & $\begin{array}{c}\hat{A} \text { ge } \\
\text { moyen }\end{array}$ & $\begin{array}{c}\text { Ancienneté moyenne } \\
\text { adhésion CFE-CGC }\end{array}$ & $\begin{array}{c}\text { Ancienneté } \\
\text { minimale }\end{array}$ & $\begin{array}{c}\text { Ancienneté } \\
\text { maximale }\end{array}$ \\
\hline Ouverture & 15 & 51 & 3,6 & 0,3 & 18 \\
Représentants du personnel & 15 & 47 & 3,8 & 0,25 & 15 \\
Élections professionnelles & 7 & 47 & 6,5 & 0,6 & 21 \\
Expression vocale et gestuelle & 10 & 45 & 7 & 2 & 28 \\
Argumentation & 14 & 47 & 6 & 3 & 24 \\
Ensemble & $61^{*}$ & 47,4 & 5 & 1,2 & 21,2 \\
\hline
\end{tabular}

* 71 stagiaires, 10 non-réponses.

Source : questionnaires (cinq formations). 
Formation et identité syndicale à la CFE-CGC

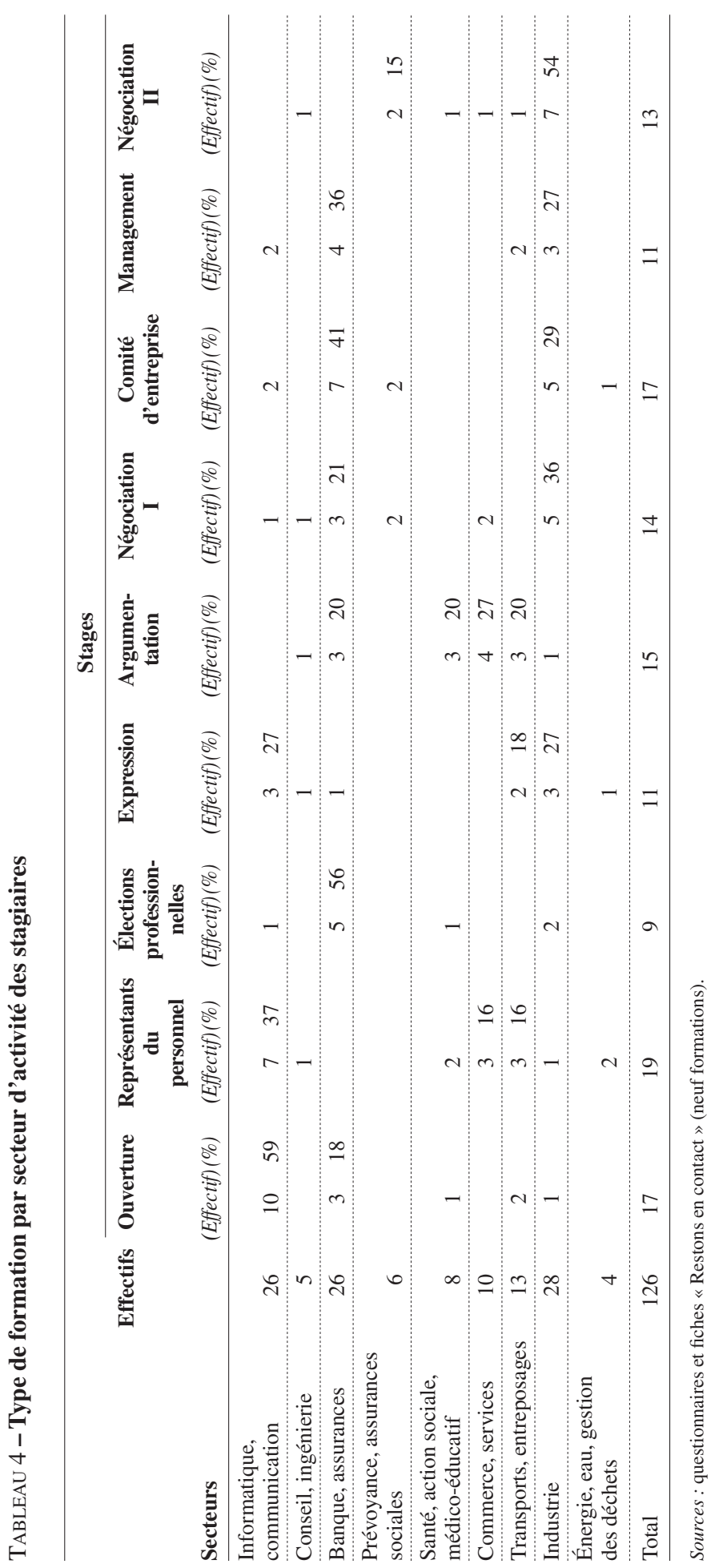

